\title{
PREPRINT
}

Version 1: $5^{\text {th }}$ June 2020

\section{Nodding off but can't disconnect: development and validation of the iNOD index of Nighttime Offline Distress}

Holly Scott ${ }^{\mathrm{a}}$, PhD; Stephany M Biello ${ }^{\mathrm{a}}$, PhD; Heather Cleland Woods ${ }^{\mathrm{a}}$, PhD

aSchool of Psychology, University of Glasgow, 62 Hillhead Street, Glasgow, G12 8QB

Corresponding author: Dr Holly Scott, holly.scott@glasgow.ac.uk

Please note: This is a preprint of a submitted manuscript. We welcome any feedback comments. 


\section{Abstract}

There is a pressing need to update sleep models, education and treatment to better reflect the realities of sleep in a 24/7 connected social world. Progress in this area has been limited to date by available measurement tools, which have largely restricted their focus to recording the frequency or duration of individuals' social media use, without capturing crucial sleep-relevant aspects of this inherently social and interactive experience. This study uses data from 3,008 adolescents (aged 10-18 years) to rigorously develop and validate a new 10-item self-report measure that quantifies the extent to which individuals struggle to disengage from social media interactions at night: the index of Nighttime Offline Distress (iNOD). The current findings indicate considerable individual differences, with most participants reporting little difficulty disconnecting but a minority experiencing unhelpful bedtime concerns around Staying Connecting and Following Etiquette to varying extents. Those with higher scores on these subscales tended to report using social media for longer after they felt they should be asleep, with shorter sleep duration and poorer sleep quality. Results also indicated that extended periods of wakefulness in bed before attempting to sleep are common amongst today's adolescents, pointing towards a potentially fragmented process of sleep displacement for those who may struggle to disconnect - and to stay disconnected - from social interactions in order to allow sufficient uninterrupted sleep opportunity. These findings can inform current models for understanding normal and disordered sleep during adolescence, whilst highlighting specific social concerns as important potential targets for sleep education efforts. The iNOD equips researchers and clinicians with a short validated self-report measure that can support efforts to move evidence and practice in this area forwards.

\section{Keywords}

Adolescence; social media; screentime; self-report measure; delayed sleep onset; sleep duration; sleep quality 
By providing adolescents with 24/7 opportunities for interaction, social media directly competes with sleep during a developmental period when the amount of sleep needed for optimal functioning remains high, but individuals are primed to prioritise peer interactions, acceptance and belonging (Blakemore \& Mills, 2014; Short et al., 2018). Social media has been highlighted as one important factor contributing to typically delayed bedtimes amongst adolescents, which - in combination with early school day rise times - make insufficient and ill-timed sleep the norm for this population (Carskadon, 2011; Crowley et al., 2018). Sleep is a key component of adolescent wellbeing, with important implications for academic performance and lifelong mental and physical health (Carskadon, 2011; Lee et al., 2014; Owens, 2014). Therefore, there is considerable current interest in the possible negative impact of social media on adolescent sleep, with concerns and calls for evidence being voiced by parents, educators, health practitioners and policymakers (The Guardian, 25 December 2016; UK House of Commons Science and Technology Committee, 2019). However, current evidence on adolescent social media use and sleep has been limited by available measurement tools, which to date have largely focused on the frequency and duration of social media use, without capturing sleeprelevant aspects of this inherently social and interactive experience. The current study builds on recent qualitative insights from an adolescent perspective (Scott et al., 2019), to rigorously develop and validate a new self-report measure that quantified the extent to which individuals struggle to disengage from social media at night: the Index of Nighttime Offline Distress (iNOD). This aims to provide a measurement tool that supports researchers and practitioners to move beyond conceptualising social media in hours per day, to build a more in-depth understanding of its unique implications for adolescent sleep.

Available literature on adolescent sleep has largely studied social media use through a techno-centric lens, grouping it together with other technology under generic umbrella terms like "screentime" or "phone use". Furthermore, studies that do examine social media specifically often continue to treat this as a technology-based activity, focusing on the frequency and duration of use (Scott \& Woods, 2019). This approach to date has provided insight into some of the mechanisms linking social media use - alongside other forms of technology - to adolescent sleep (Bartel \& Gradisar, 2017; Cain \& Gradisar, 2010). For example, an hour spent using social media is an hour spent awake, and with 
only 24 hours in the day, social media use is one of many activities that can contribute to sleep displacement (Exelmans, Gradisar, \& Van den Bulck, 2018; Van den Bulck, 2004). However, this techno-centric approach fails to capture the unique social, emotional and cognitive aspects of social media use that are specifically relevant to adolescent sleep, beyond simply another hour of total daily "screentime". An updated approach to social media interactions is needed moving forward to ensure that adolescent sleep research, education and treatment remain fit for purpose in a rapidly evolving social media landscape (Scott \& Woods, 2019).

Recent work has explored social media's unique implications for adolescent sleep, by providing an in-depth insider perspective on the experience of balancing sleep and online interactions whilst navigating adolescence (Scott et al., 2019). These qualitative findings highlighted the powerful motivations that lead some adolescents to prioritise social media over sleep, often struggling to disengage despite acknowledging sleepiness and impaired daytime functioning. These drivers included concerns about missing out on social media interactions at night - with negative consequences for "real world" peer relationships the next day - and perceived social expectations of online availability. Adolescents framed social media not as a form of technology, but as an extension of faceto-face interactions, underpinned by the same drivers, such as a desire to feel included and to follow group norms (Assunção \& Matos, 2014; Hogg \& Reid, 2006; Sheldon \& Bettencourt, 2002). Given the heightened sensitivity to social cues during this developmental period, it is perhaps unsurprising that the adolescent 'social brain' would prioritise the opportunity for peer acceptance and belonging via online interactions over the need for sufficient sleep (Blakemore, 2018; Blakemore \& Mills, 2014). This insight can help ensure that adolescent sleep models and intervention strategies remain up to date with the current challenges that adolescents themselves identify as important. This requires appropriate measurement tools that capture not simply time spent on social media but also these underlying concerns about disengaging at night.

Researchers and practitioners wishing to explore these uniquely relevant aspects of social media experiences for sleep are currently limited by the measurement tools available to them. Much of the existing literature relies on unvalidated single-item measures of frequency and duration of social media use (Jenkins-Guarnieri et al., 2013; Seabrook et al., 2016). Furthermore, available multi-item measures are often specific to one platform 
and its features, meaning that they can quickly become outmoded (The Lancet, 2019). For example, given its prevalence in the past decade, several available measures are specific to Facebook (Andreassen et al., 2012; Ellison, Steinfield, \& Lampe, 2007; Ross et al., 2009), which has now been overtaken amongst adolescents by image-based platforms Snapchat and Instagram (Pew Research Center, May 2018), whose features facilitate qualitatively different social interactions and user experiences (Pittman \& Reich, 2016). Maintaining pace with current platforms and features is an inevitable challenge in this field, but perhaps more worrying is the tendency for available measures to pathologise social media use, aiming to capture social media "disorder" or "addiction", despite insufficient evidence for considering it as such (Andreassen et al., 2012; Holmgren \& Coyne, 2017; van den Eijnden et al., 2016). Existing validated measures of fear of missing out (Przybylski et al., 2013) and emotional connection to social media use (JenkinsGuarnieri et al., 2013) have proved useful to date in starting to examine the cognitivebehavioural aspects of social media use that are particularly relevant for sleep (Scott \& Woods, 2018; Woods \& Scott, 2016). To move forward, a targeted sleep-specific measure is now required that targets the concerns that adolescents themselves identify as causing difficulties disengaging from social media at night.

To date, the choice of available measures has limited the scope of research questions, conclusions, recommendations and wider dialogue, maintaining a focus on time spent using social media, which is often framed negatively (The Guardian, 25 December 2016; UK House of Commons Science and Technology Committee, 2019). This study targets this gap by developing and validating a new self-report measure of difficulty disengaging from social media at night due to underlying concerns, which is crucial to draw conclusions about the sleep-specific implications of adolescent social media use. Drawing on recent qualitative findings, this study first develops data-driven candidate items whose content and wording capture the concerns that adolescents identify as driving nighttime social media habits that impact on sleep (Scott et al., 2019). It uses data from a large adolescent sample to select items for the final measure - the Index of Nighttime Offline Distress (iNOD) - and to examine its factor structure, validity and reliability. Finally, we explore to what extent individuals differ on this difficulty disengaging construct, and how it is associated with nighttime social media habits and a range of sleep measures. Based on literature to date, we expect that adolescents who experience these concerns more 
strongly will tend to use social media for longer in bed and after the time they feel they should be asleep, and report later bedtimes, shorter sleep duration and poorer sleep quality.

\subsection{Methods}

\subsubsection{Participants and procedure}

Participants were 3008 adolescents attending Scottish secondary schools (51.1\% female), aged 10-18 years old (mean age $=14.6$ years). This online survey study was advertised online to recruit partner schools. Each school received a tailored summary of typical sleep and social media habits in their school, to support evidence-based school policy and curriculum development. Each school invited its pupils to complete the online survey: often this was the whole school, or in some cases only certain year groups took part based on their curriculum and availability. Participating pupils completed the online survey, which included candidate items for the new measure and other established questionnaire measures (see Measures, section 1.1.2). Most schools invited pupils to participate during classes, while a few schools invited pupils to complete the survey using their own devices outside of class times. Average completion time was 15 minutes.

Parents received advance information on the study, with the option to opt-out their child. The first page of the online survey provided a plain English summary of pupils' rights to non-participation and confidentiality, with the researcher contact details. Pupils had to indicate consent via a tick box before proceeding to the survey. This study was approved by the University of Glasgow College of Science and Engineering Ethics Committee, a senior staff member from each participating school, and relevant local authorities where research approval procedures were in place. Partner schools and participating pupils did not receive monetary reimbursement.

\subsubsection{Measures}

Participants reported their date of birth and sex and completed the candidate items (details below). The survey also included existing measures that capture related constructs to test convergent and discriminant validity: emotional connection to social media, fear of missing out and personality traits. Participants also reported on typical 
sleep patterns and completed a measure of sleep quality. Details of each measure are provided below.

\subsubsection{1 iNOD candidate items}

We developed candidate items for the new measure based on the theme structure identified in the focus group thematic analysis in the previous chapter. Table 1 presents the candidate items, according to the sub-themes they were intended to capture. These items were discussed amongst the research team and in consultation with three external researchers with expertise in media engagement (two of whom had additional expertise in sleep and one of whom had additional expertise in scale item generation). This process refined the content and wording of candidate items to ensure they captured the intended construct. We included deliberate redundancy with multiple wordings of similar underlying concepts (DeVellis, 2016).

The questionnaire instructions were: "Thinking about how you use social media at night, please rate each of the following statements from 'not at all true of me' to 'extremely true of me'." The candidate items were then listed in randomised order. The response options were: "not at all true of me" (0), "slightly true of me" (1), "moderately true of me" (2), "very true of me" (3) and "extremely true of me" (4). 


\begin{tabular}{ll}
\hline Theme & Sub-theme \\
\hline Missing Out & Offline costs
\end{tabular}

Perceived negative impact on offline relationships (e.g. peer exclusion) from missing social media content \& interactions

\section{Constant threat}

State of threat/unpleasantness when offline: intrusive thoughts \& bedtime rumination about possibly missing out

\section{Candidate items}

1. I worry that I might miss something that my friends have seen on social media

2. I worry that I will be left out from my friends the next day if I don't see something on social media that night

3. It's important to me that I've seen the same things on social media as my friends

4. I would feel left out from my friends if I couldn't use social media at night

5. When I'm not using social media, I feel like I don't know what's going on

6. I feel like I'm missing out on something if I'm not on social media around bedtime

7. It's always in the back of my head when I'm not on social media

8. If I'm not on social media around bedtime, I wonder if my friends are

9. When I'm trying to fall asleep, I think about social media

\section{Can't disengage}

Difficulties disengaging from social media (\& resisting temptation to re-engage with incoming notifications / new content)

10. I need to be up to date with things on social media before I can relax at bedtime

11. I feel like I have to check my account(s) for anything new before I can sleep

12. I listen/look out for notifications while l'm trying to sleep

13. It's important for me to check notifications and new content before I go to sleep

14. I would find it difficult not to check a notification when I'm trying to sleep

\begin{tabular}{ll}
\hline Social & Normal behaviours \\
Expectations & Perceived 'normal' or typical teenager/peer social \\
& media habits (available at night, prompt responses)
\end{tabular}

15. I feel like my friends expect me to be on social media around my bedtime

16. I feel like my friends expect me to be on social media throughout the night

17. I feel like my friends expect me to answer messages quickly

18. I feel like my friends expect me to have seen the latest things on social media

19. I feel like normal people my age use social media late at night

20. I worry that I will offend someone if I leave a conversation

21. I feel like a bad friend if I don't answer a message quickly

Feeling of guilt / being rude when not responding

22. If I don't answer a message quickly, I feel rude

23. If I end a conversation, I feel like a bad friend

24. I feel rude if I end a conversation

25. I feel like I'm letting my friends down if they can't contact me on social media

\section{Obligation}

Continuing online interactions out of obligation, not

26. I feel like I have to answer messages even if I'm trying to sleep

choice (i.e. when tired but 'stuck talking to someone')

27. I feel like I have to continue a conversation even if I'm tired and want to fall asleep

28. I feel like I have to respond to things on social media even if I'm sleepy and don't feel like it 


\subsubsection{Emotional connection to social media}

We used the Social Integration and Emotional Connection subscale of the Social Media Use Integration Scale (Jenkins-Guarnieri et al., 2013), slightly modified by replacing the original "Facebook" with "social media" in the item wordings. Items included "I get upset when I can't log on to social media" and were rated on a 6-point Likert scale from "strongly disagree" to "strongly agree". Items scores were summed to give a total subscale score of 6-36, with higher scores indicating higher levels of emotional connection to social media. The measure had good reliability in the current sample (Cronbach's alpha $=.84)$.

\subsubsection{Fear of missing out}

The Fear of Missing Out scale captures the extent to which an individual experiences a "pervasive apprehension that others might be having rewarding experiences from which one is absent" (Przybylski et al., 2013, p. 1841). It consists of 10 items, such as "I get anxious when I don't know what my friends are up to", which are rated on a 5-point scale from "not at all true of me" to "extremely true of me". The overall score (from 1-5) is calculated by averaging all 10 item scores. The scale had good reliability in the current sample (Cronbach's alpha $=.89)$.

\subsubsection{Big Five personality traits}

To assess the discriminant validity of the new measure, we used the mini International Personality Item Pool (mini-IPIP; Donnellan, Oswald, Baird, \& Lucas, 2006) to measure the Big Five personality traits: Extraversion, Agreeableness, Openness, Neuroticism and Conscientiousness. Each four-item measure had acceptable reliability in the current sample (Cronbach's alphas $>=.67)$.

\subsubsection{Nighttime social media use}

Participants provided estimates of how long (in hours and minutes) they typically use social media in bed at night, and after they feel they should be sleeping. 


\subsubsection{Sleep parameters}

Participants reported the times at which they typically went to bed, and closed their eyes to sleep ("shuteye time"; Exelmans \& Van den Bulck, 2017a). They also provided estimates of their typical total sleep duration, not counting any periods of wake during the night.

\subsubsection{Sleep quality}

We used the Sleep Condition Indicator (SCl; Espie et al., 2014) to measure sleep quality. It has eight items that capture difficulties initiating and maintaining sleep, perceived sleep quality and daytime functioning, and the duration and frequency of sleep problems. Items are scored out of 4 and summed to give a total score between 0 and 32, with lower scores indicating poorer sleep quality. Scores of 16 or below are used to indicate possible insomnia. It had good reliability in the current sample (Cronbach's alpha $=.83$ ).

\subsubsection{Data analysis}

Data analysis was completed using $\mathrm{R}$ version 3.5.1 (R Core Team, 2018). We randomly split the candidate item data into a calibration sample and a holdout sample (DeVellis, 2016).

The calibration sample was submitted to Exploratory Factor Analysis (EFA) using maximum likelihood extraction and promax (oblique) rotation. To identify the most appropriate number of factors to extract, we consulted multiple criteria including: Eigenvalues > 1 (Kaiser, 1960); the inflection point from a visual inspection of the scree plot (Cattell, 1966); adjusted Eigenvalues > 0 from parallel analysis (Glorfeld, 1995; Horn, 1965); and the interpretability of resulting factor solutions (Jenkins-Guarnieri et al., 2013). After extracting factors, we retained items with primary factor loadings $>=.5$ and secondary factor loadings <.3, to produce a clean factor structure. We then reduced the number of items by considering the content, wording and performance of items in the factor solution (DeVellis, 2016). We re-examined the factor structure of this final solution and calculated Cronbach's alpha to assess its reliability (alpha > .7 is "good", >.8 is "very good", and > .9 is "excellent"; P. Kline, 2000). 
The holdout sample was submitted to Confirmatory Factor Analysis to test the fit of this model. We computed a mean- and variance- adjusted Diagonal Weighted Least Squares statistic with robust standard errors (Rosseel, 2012). The following criteria were used to determine acceptable model fit: comparative fit index (CFI) and Tucker-Lewis index (TLI) $>.90$ (Hu \& Bentler, 1999); and root-mean-square error of approximation (RMSEA) and standardised root mean square residual $(S R M R)<.08$ (M. W. Browne \& Cudeck, 1992; R. Kline, 2005). A significant Chi-square value is used to indicate poor model fit in small samples (R. Kline, 2005), but this is common in larger samples and can be disregarded when $\mathrm{n}>200$ (Hair, Tatham, Anderson, \& Black, 1998).

We examined the properties of the final measure using data from the whole sample together. We tested age differences with Spearman's correlation coefficients and gender differences with Yuen's bootstrap t-test (a robust alternative to standard independent samples t-test; Wilcox, 2012). Convergent and discriminant validity (Hubley, 2014) were tested using Spearman's correlations between the final measure and existing measures that should show medium to large associations (fear of missing out, emotional connection to social media, nighttime social media use) or small to no associations (the Big Five personality traits). Finally, we examined associations between the new measure and nighttime social media habits and sleep measures, first using Spearman's correlations and then using multiple regressions that controlled for age and gender.

\subsection{Results}

We first examined the distributions of each item and removed items 9 and 12 due to unacceptable skew and kurtosis, respectively. The remaining 26 items all had skew and kurtosis within the acceptable range (between -2 and +2; Gravetter \& Wallnau, 2014). There were complete case data for these 26 items from 2866 participants. These were randomly split into a calibration and a test sample, each with 1433 participants (an “excellent” sample size for factor analysis; Comfrey and Lee, 1992).

\subsubsection{Calibration dataset: Exploratory Factor Analysis \& Item Analysis}

The factorability of the calibration dataset was supported by Bartlett's Test of Sphericity $(p<.001)$ and the Kaiser-Meyer-Olkin measure (KMO = .970; Kaiser, 1974; Watson, 2017). 
We considered the multiple criteria outlined in the Data Analysis section for identifying the number of factors to extract. Different criteria suggested different numbers of factors, so we examined the interpretability of each factor solution and established that extracting two factors (with Eigenvalues of 12.44 and 1.23 respectively) produced the most meaningful solution. This was consistent with the Kaiser rule of retaining those factors that have Eigenvalues $>1$ (Kaiser, 1960).

We performed EFA to extract two factors and considered the resulting loading patterns against criteria in Data Analysis section. This resulted in 20 of these 26 items being retained. Items 17, 19, 25, 26, 27 and 28 were all discarded due to primary factor loadings $<0.5$. The resulting 20 -item two-factor solution was stable and did not change when subjected to another iteration of EFA. This produced a clean and interpretable factor structure. Table 2 shows the factor loadings for the 20-item two-factor solution. 
6 I feel like I am missing out on something if I am not on social media around bedtime

10 I need to be up to date with things on social media before I can relax at bedtime

$\mathbf{0 . 7 7 6}-0.060$

4 I would feel left out from my friends if I could not use social media at night

$\mathbf{0 . 7 7 5}-0.035$

11 I feel like I have to check my accounts for anything new before I can sleep

5 When I am not using social media, I feel like I do not know what is going on

3 It is important to me that I have seen the same things on social media as my friends

13 It is important for me to check notifications and new content before I go to sleep

1 I worry that I might miss something that my friends have seen on social media

7 It is always in the back of my head when I am not on social media

$0.699 \quad 0.036$

2 I worry that I will be left out from my friends the next day if I do not see something on social media that night

15 I feel like my friends expect me to be on social media around my bedtime

8 If I am not on social media around bedtime, I wonder if my friends are

18 I feel like my friends expect me to have seen the latest things on social media

16 I feel like my friends expect me to be on social media throughout the night

14 I would find it difficult not to check a notification when I am trying to sleep

22 If I do not answer a message quickly, I feel rude

21 I feel like a bad friend if I do not answer a message quickly

20 I worry that I will offend someone if I leave a conversation 
Factor 1 (15 items) and factor 2 ( 5 items) had Cronbach's alphas of .94 and .93, respectively. Any Cronbach's alpha above .9 is considered excellent (Kline, 2000). We therefore considered whether the number of items could be reduced whilst retaining good reliability. This aimed to produce the most useful final measure by balancing strong reliability with minimal respondent burden (DeVellis, 2016). We considered the conceptual consistency of item content, the length of item wordings and how each item performed in item analysis. For factor 1, we removed items 1, 3, 5, 7 and 18, whose wordings did not specifically mention bedtime or sleep, to maximise consistency of the intended construct. We removed item 2, whose wording was considerably longer than other items, to optimise readability. The remaining ten items had an unchanged Cronbach's alpha value of .94. We then iteratively removed items with the lowest itemtotal correlation, to produce a final six-item subscale which retained excellent reliability (Cronbach's alpha $=.91)$. For factor 2 , we removed item 22 - since it had the lowest item-total correlation - to produce a final four-item subscale, which also had excellent reliability (Cronbach's alpha $=.92)$.

We once again performed EFA to these ten items, which retained a clean factor structure. The factor loadings for this 10-item two-factor solution are shown in Table 3. Factor 1 explained $32.2 \%$ of the variance. Factor 2 explained $25.0 \%$ of the variance. 


\begin{tabular}{|c|c|c|c|}
\hline \multirow[b]{2}{*}{ Item } & \multirow[b]{2}{*}{ Item text } & \multicolumn{2}{|c|}{ Factor loadings } \\
\hline & & Factor 1 & Factor 2 \\
\hline 6 & $\begin{array}{l}\text { I feel like I am missing out on something if I am not on social } \\
\text { media around bedtime }\end{array}$ & 0.830 & -0.062 \\
\hline 4 & $\begin{array}{l}\text { I would feel left out from my friends if I could not use social } \\
\text { media at night }\end{array}$ & 0.790 & -0.041 \\
\hline 15 & $\begin{array}{l}\text { I feel like my friends expect me to be on social media around } \\
\text { my bedtime }\end{array}$ & 0.732 & -0.005 \\
\hline 16 & $\begin{array}{l}\text { I feel like my friends expect me to be on social media } \\
\text { throughout the night }\end{array}$ & 0.674 & 0.071 \\
\hline 10 & $\begin{array}{l}\text { I need to be up to date with things on social media before I } \\
\text { can relax at bedtime }\end{array}$ & 0.669 & 0.019 \\
\hline 8 & $\begin{array}{l}\text { If I am not on social media around bedtime, I wonder if my } \\
\text { friends are }\end{array}$ & 0.661 & 0.089 \\
\hline 21 & I feel like a bad friend if I do not answer a message quickly & 0.129 & 0.669 \\
\hline 20 & I worry that I will offend someone if I leave a conversation & 0.091 & 0.715 \\
\hline 23 & If I end a conversation, I feel like a bad friend & -0.030 & 0.901 \\
\hline 24 & I feel rude if I end a conversation & -0.039 & 0.842 \\
\hline
\end{tabular}

\subsubsection{Holdout sample: Confirmatory Factor Analysis}

We then performed Confirmatory Factor Analysis of the 10-item two-factor model in the holdout sample of 1433 participants. The chi-square test was significant $\left(X^{2}[34]=307.67\right.$, $p<.001$ ), although this is common in large samples (Hair et al., 1998). The remaining goodness of fit indices met the criteria outlined in the Data Analysis section, supporting acceptable fit of the model $(\mathrm{CFI}=.984, \mathrm{TLI}=.979, \mathrm{RMSEA}=.075, \mathrm{SRMR} .031)$.

Both subscales had excellent reliability in the holdout sample, with Cronbach's alpha of .91 for factor 1 and .92 for factor 2. Based on the item content for each factor, we named factor 1 Staying Connected and factor 2 Following Etiquette. 


\subsubsection{Final measure: correlations and distribution}

The remaining analyses examined the properties of the final subscale scores and how they related to other measures. For these remaining analyses, we used data from the whole sample with pairwise deletion.

Table 4 - Means and sex differences for all measures

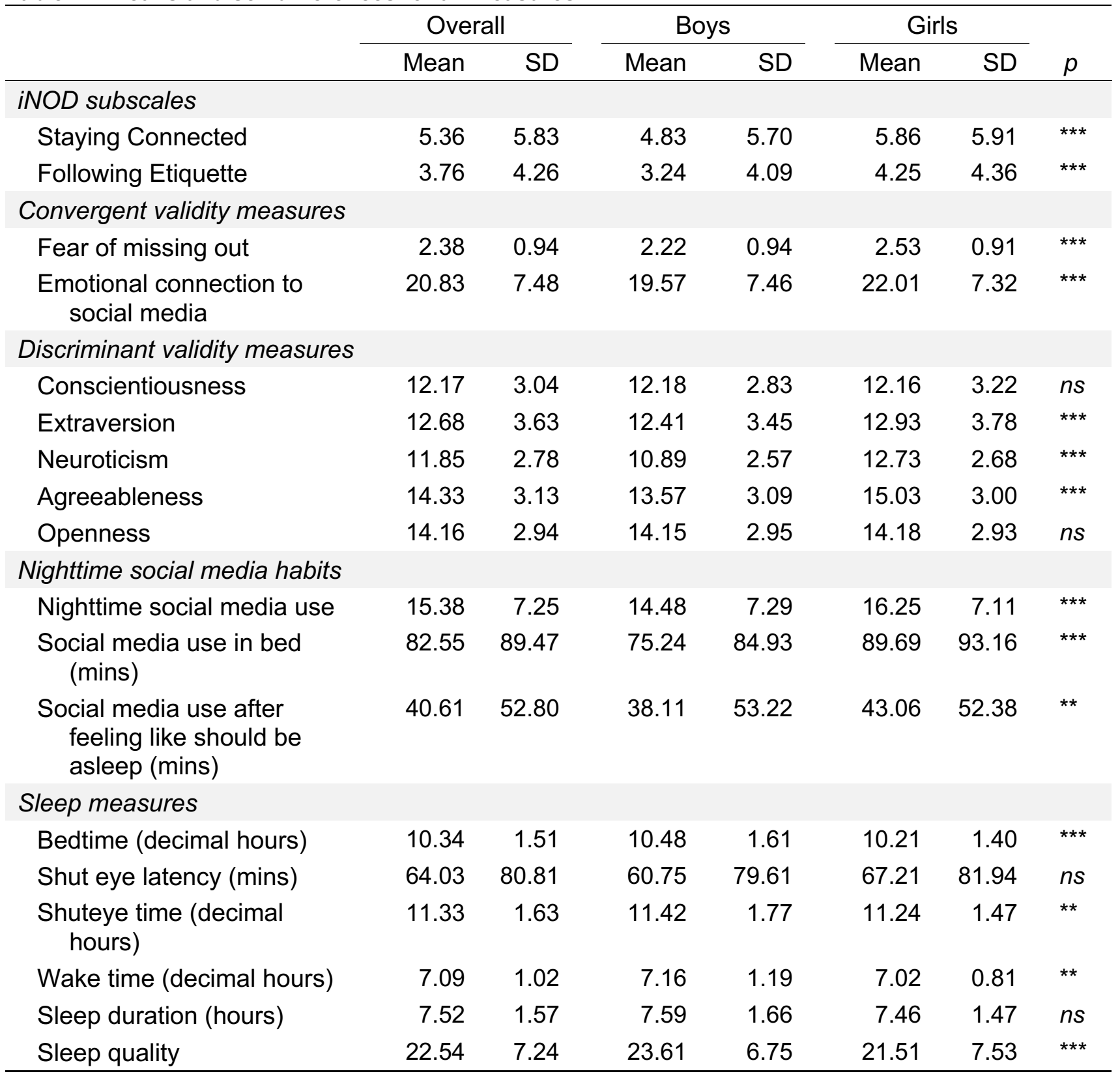

Notes: Sex differences tested by Yuen's bootstrap t-test (1000 bootstrap iterations), ${ }^{* *} p<.01,{ }^{* * *} p$ $<.001$. 


\subsubsection{Demographic associations}

Table 4 presents means and standard deviations for all measures, including a breakdown by sex (with significant sex differences tested by Yuen's bootstrap t-test). Girls scored higher on both iNOD subscales, fear of missing out and emotional connection to social media. Girls also tended to use social media for longer in bed and after the time they felt they should be asleep. There was no gender difference in sleep duration, although boys tended to go to bed and wake up later than girls. Girls had poorer sleep quality than boys.

Age was not significantly associated with Staying Connected $\left(r_{s}=.01, p=.454\right)$. There was a very small but statistically significant association between age and Following Etiquette $\left(r_{s}=.06, p<.01\right)$. Compared to younger adolescents, older adolescents tended to go to bed later $\left(r_{s}=.18, \mathrm{p}<.001\right)$, but wake up at the same time $\left(r_{s}=.04, \mathrm{p}=0.053\right)$, therefore reporting shorter sleep duration $\left(r_{s}=-.21, \mathrm{p}<.001\right)$. Older adolescents also tended to use social media for longer in bed $\left(r_{s}=.14, \mathrm{p}<.001\right)$ and after the time they felt they should be asleep $\left(r_{s}=.14, \mathrm{p}<.001\right)$.

Although skew statistics for both subscales were within an acceptable range (1.27 for Staying Connected subscale and 1.24 for Following Etiquette subscale; Gravetter \& Wallnau, 2014), visual inspection of their distributions identified a positive skew (see Figure 1). There was a floor effect on both subscales, with considerable percentages of participants scoring zero on Staying Connected (24.1\%) and Following Etiquette (30.4\%).

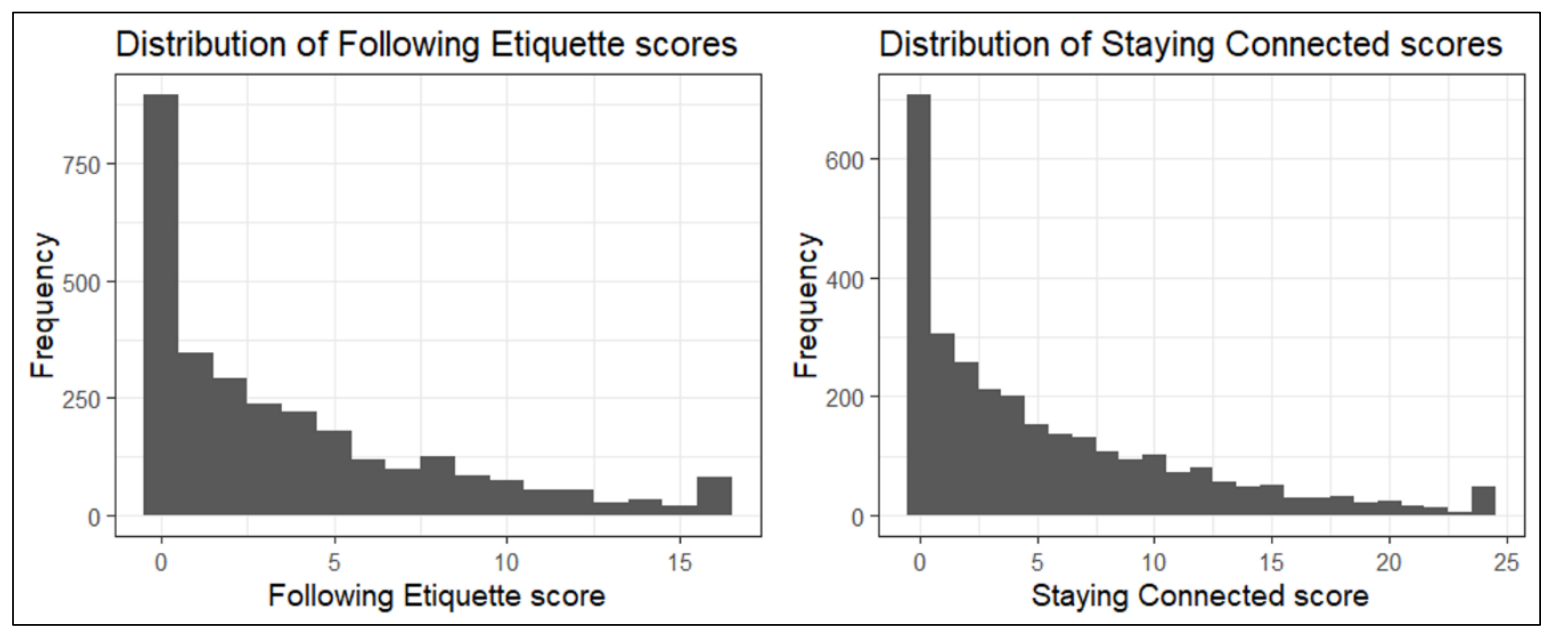

Figure 1 - Distributions of iNOD subscale total scores 


\subsubsection{Convergent and discriminant validity}

Both subscales showed patterns of convergent and discriminant validity consistent with the intended construct (see Table 5 for correlations). For convergent validity, the iNOD subscales had medium to large correlations with related measures of emotional connection to social media, fear of missing out and nighttime social media use. For discriminant validity, the iNOD subscales had smaller (or no) correlations with the Big Five personality traits (highest $r_{s}=.23$ ), and the effect sizes of these personality associations were comparable for existing validated measures (FoMOS and SMUIS).

Table 5 - iNOD subscale correlations with related measures and personality traits

\begin{tabular}{|c|c|c|c|c|c|}
\hline & 1 & 2 & 3 & 4 & 5 \\
\hline 1. iNOD 1 : Staying Connected & - & & & & \\
\hline 2. iNOD 2 : Following Etiquette & $.59^{* * *}$ & - & & & \\
\hline 3. Fear of missing out & $.63^{* * *}$ & $.53^{* * *}$ & - & & \\
\hline 4. Emotional connection to social media & $.59^{* * *}$ & $.43^{* * *}$ & $.57^{* * *}$ & - & \\
\hline 5. Nighttime social media use & $.47^{* * *}$ & $.30^{* * *}$ & $.37^{* * *}$ & $.46^{* * *}$ & - \\
\hline 6. Conscientiousness & $-.17^{\star \star \star}$ & $-.15^{\star * *}$ & $-.16^{\star \star \star}$ & $-.20^{\star \star *}$ & $-.26^{\star * *}$ \\
\hline 7. Extraversion & $.12^{* * *}$ & ns & $.11^{* \star *}$ & $.15^{\star * *}$ & $.17^{* * *}$ \\
\hline 8. Neuroticism & $.23^{* * *}$ & $.22^{* * *}$ & $.29^{\star \star *}$ & $.22^{* * *}$ & $.20^{* * *}$ \\
\hline 9. Agreeableness & $.06^{* *}$ & $.14^{* * *}$ & $.18^{* * *}$ & $.11^{* * *}$ & ns \\
\hline 10. Openness & $-.19^{\star \star *}$ & $-.15^{\star * *}$ & $-.13^{\star \star *}$ & $-.19^{\star \star \star}$ & $-.20^{\star \star \star}$ \\
\hline
\end{tabular}

Notes: Spearman correlation coefficients. After pairwise deletion, sample size $n$ for individual correlations ranges from 2570 to $2948 .{ }^{* *} p<.01,{ }^{* * *} p<.001, n s=$ non-significant. Significance levels calculated using Holm-Bonferroni correction for multiple comparisons (Haynes, 2013; Holm, 1979).

\subsubsection{Associations with nighttime social media use and sleep measures}

Table 6 shows correlations between the iNOD subscales, bedtime social media habits, sleep patterns and sleep quality. Those who scored higher on Staying Connected and Following Etiquette tended to get into bed later, have longer shut eye latency and later shuteye times, but did not differ in their wake times. They also tended to use social media for longer in bed and after the time they felt they should be asleep. They tended to have shorter sleep duration and poorer sleep quality. Staying Connected showed stronger associations with bedtime social media habits and sleep measures than Following Etiquette. These associations remained significant in multiple regression models that controlled for age and sex. 


$\begin{array}{lllllllll}1 & 2 & 3 & 4 & 5 & 6 & 7 & 8 & 9\end{array}$

1. iNOD Staying Connected

2. iNOD Following Etiquette

$-$

3. Bedtime

4. Shut eye latency

5. Shuteye time

6. Wake time

$.59^{* * *}$

$.16^{* * *} \cdot .11^{* * *} \quad-$

$.14^{* * *} \quad .07^{* * *}-.30^{* * *}-$

$.28^{* * *} \quad .17^{* * *} \quad .58^{* * *} \quad .46^{* * *}-$

ns $\quad$ ns $\quad .21^{* * *} \quad .07^{* * *} \quad .23^{* * *}$ -

7. Social media use in bed

$\begin{array}{llllll}.37^{* * *} & .20^{* * *} & .20^{* * *} & .37^{* * *} & .52^{* * *} & .12^{* * *}\end{array}$

8. Social media use after feeling like should be asleep

9. Sleep duration

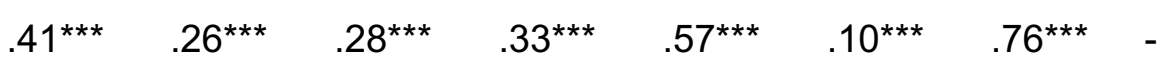

$\begin{array}{llllllll}-.24^{* * *} & -.17^{* * *} & -.34^{* * *} & -.28^{* * *} & -.58^{* * *} & .18^{* * *} & -.38^{* * *} & -.44^{* * *}\end{array}$

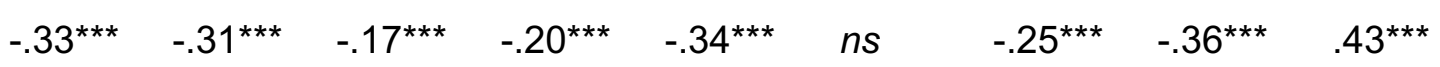

Notes: Spearman's correlation coefficients. All reported coefficients in this table are significant at $p<.001$. Significance levels calculated using Holm-Bonferroni correction for multiple comparisons (Haynes, 2013; Holm, 1979). 



\subsection{Discussion}

This study developed a new data-driven, validated self-report measure of difficulty disengaging from social media at night: the index of Nighttime Offline Distress (iNOD). The final 10-item measure has two subscales with excellent reliability that capture concerns about Staying Connected to peers via social media at night and Following Etiquette by continuing ongoing interactions at night. The iNOD moves beyond existing measures to capture a unique construct that is highly relevant given current interest in better understanding and supporting adolescent sleep in an evolving social media context. It provides a rigorously developed tool that is informed by the adolescent voice (Scott et al., 2019) and allows researchers and clinicians to target these core underlying concerns to explore social media's unique implications for adolescent sleep. In the current study, individuals who experienced these concerns more strongly also tended to use social media for longer in bed, and reported later shuteye times, shorter sleep duration and poorer sleep quality.

\subsubsection{A new measurement tool: a unique construct \& contribution}

The iNOD provides a new tool that targets the sleep-specific issues around social media that are highly relevant to today's adolescents, by using recent qualitative findings from young people as the foundation for its constructs and items (Scott et al., 2019). The Staying Connected subscale measures concern over remaining connected to friends via social media at night, to avoid missing out on online interactions. In the previous qualitative findings, adolescents valued the social connection that social media afforded them at night: "so that you feel like you are part of something" (Scott et al., 2019). Consistent with a cognitive-behavioural perspective on nighttime social media use (Scott \& Woods, 2018), adolescents voiced how this concern about being absent from online interactions could interfere with efforts to relax at bedtime once disconnected: "you're always wondering 'what's everyone else doing? Are they speaking to each other? Am I missing out? Should I be on this? Should I be up?" (Scott et al., 2019). The Following Etiquette subscale measures concern about causing offence or appearing rude by not responding and continuing nighttime social media interactions. Previous qualitative findings highlighted this shared perception of expected social media etiquette: "if the conversation is going good you need to keep it going, you can't ignore them or else that's 
just rude" (Scott et al., 2019). This could leave some adolescents struggling to disengage from ongoing interactions at night, beyond intended bedtimes: "you don't want to offend the other person [...] so you sort of get stuck talking to a person".

The iNOD therefore offers more in-depth insight into adolescent sleep and social media use, allowing researchers to move beyond the prevalent current focus on simply time spent using devices. Indeed, the current patterns of convergent and discriminant validity support the uniqueness of its construct. Moderate to large correlations indicated that adolescents who were more concerned about Staying Connected and Following Etiquette did also experience stronger overall fear of missing out and general emotional connection to social media, but that these measures did not fully overlap. Smaller associations between the new subscales and personality traits indicated that the iNOD does not simply tap into more generalised underlying levels of concern (e.g. neuroticism), but specifically captures the more targeted intended construct of concerns about disengaging from social media at night. This novel sleep-specific measure can therefore provide new understanding of the nature of distress and unease that some adolescents can experience when disconnecting from social media at night, which is a key issue for understanding and supporting adolescent sleep in today's connected world.

This fills a gap in previously available measurement tools, which to date have limited the scope of research questions and conclusions, and therefore evidence-based decisionmaking in policy and practice. With existing measures often focusing on the frequency and duration of social media use, or apparently "addictive" symptoms, this has limited scientific understanding and wider public dialogue by missing insight into other potentially relevant aspects of social media use. The iNOD addresses this gap within the context of adolescent sleep, capturing specifically sleep-relevant aspects of this inherently social and interactive experience. This allows researchers and practitioners to move beyond the current focus on "screentime" to examine the unique processes of sleep as an adolescent in a 24/7 connected world. By addressing this gap, the iNOD can help support efforts to tackle several challenges currently facing this field (for a review see Scott \& Woods, 2019), as outlined below.

Firstly, it provides a measure with better longevity than platform- or feature- specific measures in a rapidly evolving social media landscape, which is especially relevant given 
calls for longitudinal work (Bhat et al., 2018). Secondly, it can support efforts to develop more effective interventions to promote healthy sleep by meaningfully targeting underlying concerns, moving beyond attempts to limit device access, which have proved challenging to implement (Bartel et al., 2019). Thirdly, by providing an alternative to measures that pathologise social media use, it supports researchers and practitioners to consider a more balanced, holistic model of social media interactions in the context of adolescent sleep and psychosocial development. Finally, it can help future efforts to build on current models of sleep and technology use generally (Bartel \& Gradisar, 2017; Cain \& Gradisar, 2010), to add new understanding of the unique implications that social media in particular has for adolescent sleep.

\subsubsection{Individual differences in difficulty disengaging}

The current distribution of iNOD scores indicated that a considerable proportion of adolescents do not struggle to disengage from social media at night, which challenges the prevalent narrative of universally highly engaged or "addicted" teenage social media users. The measure is therefore useful to distinguish the smaller proportion of adolescents who do experience moderate to high levels of concerns about disengaging, and to quantify the extent of their difficulty. To minimise floor effects that simply reflected the characteristics of item wordings or response options (DeVellis, 2016), we avoided generating candidate items with overly strong or negative wordings (e.g. "I get upset about...", “I struggle with...”), and used response options consistent with existing measures (Przybylski et al., 2013). Therefore, the current distribution is less likely to be an artefact of the item or response wordings, and more likely to indicate that a majority of adolescents genuinely do not find disengaging from social media particularly problematic, but that a smaller proportion do struggle with this, to varying extents.

It is also possible that some low iNOD scorers were simply under-reporting the extent to which they experience these concerns. This possibility would be consistent with previous apparently contradictory self-reports from adolescents who did describe examples of social media affecting their sleep but did not explicitly label this as such when asked directly whether social media affects their sleep (Scott et al., 2019). This is an inevitable challenge for all self-report measures, and highlights the value of triangulating subjective and objective insight into nighttime social media use and sleep. However, we stress the 
importance of understanding this subjective experience, even where it diverges from objective measures. For example, when supporting adolescents to implement healthier nighttime social media habits, the extent to which they perceive their social media use to be problematic will be an important factor in determining their level of motivation to change, which is a key component for the success of youth sleep interventions (Cain, Gradisar, \& Moseley, 2011). Therefore, the iNOD may present a useful screening tool for practitioners to capture the extent to which an individual identifies social media as a source of sleep disturbance and also to start a dialogue around these underlying concerns, for example as part of a motivational interviewing approach that focuses on promoting autonomy (Cassoff, Rushani, Gruber, \& Knäuper, 2014).

\subsubsection{New understanding of sleep models}

Beyond providing a new measurement tool that can support future research and practice, the current findings also have more immediate implications for our understanding of social media use and sleep in adolescence. Scores on both Staying Connected and Following Etiquette were associated with more time using social media in bed (including after intended bedtimes), later shuteye times, shorter sleep duration and poorer sleep quality. These findings build on existing models of general technology use and adolescent sleep (Bartel \& Gradisar, 2017; Cain \& Gradisar, 2010), to add additional understanding for social media specifically that takes into account its unique social and interactive affordances (Carr \& Hayes, 2015). Specifically, this can inform Cain and Gradisar's (2010) existing model - updated in 2017 by Bartel and Gradisar - by (1) adding to the identified factors that moderate sleep-relevant technology use (e.g. parental involvement) and (2) refining the possible mechanisms linking it to poor sleep (e.g. increased arousal). Firstly, it is now clear that an important factor in determining sleep-relevant social media behaviour is the extent to which an individual is concerned about the negative consequences of disengaging at night, whether for losing social connection or violating perceived etiquette. Secondly, we now have novel understanding of the nature of increased arousal that may link social media use to poor sleep, with a strong social influence on individuals' cognitive-emotional response to bedtime social media interactions. Together, these new insights can guide efforts to develop effective sleep intervention and education strategies, which should aim to address these social, emotional and cognitive aspects of bedtime social media use in order to remain fit for 
purpose in the changing sleep environment of today's adolescents. This builds on a cognitive-behavioural perspective on adolescent bedtime social media use and sleep, which suggests that interventions focusing purely on reducing time spent using social media are not only challenging to implement (Bartel et al., 2019), but also fail to address the parallel influence of cognitive arousal from potential distress of being disconnected (Scott \& Woods, 2018).

The current findings also support the need to update our thinking about sleep in general, to account for the way in which bedtime media use has altered the typical sleep environment and processes. Exelmans \& Van den Bulck (2017) recently argued for sleep displacement to be recharacterised as a two stage process, with media activities such as social media use not only delaying the time individuals get into bed (stage 1 sleep displacement) but also extending the time spent awake in bed before actually deciding to go to sleep (stage 2 sleep displacement). They introduced the concept of shuteye latency, as the time between getting into bed and actually closing one's eyes and deciding to sleep. In the current adolescent sample, this shuteye latency was on average 1 hour 4 minutes, which is roughly 20 minutes longer than previously noted in a representative adult sample (Exelmans \& Van den Bulck, 2017a). This reinforces the importance of clearly defining whether research measures and findings apply to bedtimes or shuteye times in today's often media-rich sleep environment, and this consideration may be even more important for adolescents. For some young people, this extended period of wakefulness in bed before attempting to sleep could reflect a mismatch between parental expectations around appropriate bedtimes and bioregulatory pressure for later sleep onset given delayed circadian rhythm and slower build-up of sleep pressure during adolescence (Carskadon, 2011; Crowley et al., 2018).

Furthermore, the current findings could indicate an even more fragmented process of sleep displacement - beyond just two consolidated stages - for adolescent social media users. Participants reported spending on average 1 hour 23 minutes using social media in bed at night, which is longer than the average shuteye latency ( 1 hour 4 minutes). This suggests additional sleep interruptions after initial shuteye times, if these adolescents were re-engaging with social media again after the time that they first closed their eyes and decided to go to sleep, consistent with previous self-reports (Scott et al., 2019). Incoming alerts and notifications may therefore further fragment the process of sleep 
displacement, by not only delaying bedtimes and initial shuteye times (Exelmans \& Van den Bulck, 2017a), but also interrupting and resetting sleep onset latency for individuals who re-engage with social media after first deciding to sleep. Indeed, on average participants reported using social media for $\mathbf{4 1}$ minutes after the time they felt they should be asleep. This is consistent with previous findings that adolescents find it difficult to follow intended self-regulation strategies to limit their nighttime use, struggling to avoid re-engaging with social media due to incoming alerts (Scott et al., 2019). These findings extend existing evidence highlighting the need to rethink and update sleep measures and models, since typical sleep habits may now involve an increasingly fragmented process of sleep displacement, facilitated by highly prevalent hand-held devices with integrated social media apps that are accessible 24/7 and push through notifications designed to re-capture users attention.

\subsubsection{Strengths \& limitations}

The strengths of this study include its large, diverse sample of adolescents from schools across Scotland and its rigorous methods for item generation and selection (DeVellis, 2016). Using self-report is a strength of the iNOD measurement approach, as this is crucial to capture adolescents' cognitive and emotional experience of difficulty disengaging from social media due to social concerns. However, when estimating social media duration or sleep patterns, the current study's reliance on self-report can be considered a limitation. This type of self-report estimate is common in the literature, but is subject to bias in recall and reporting (Althubaiti, 2016), for example with sleep state misperception resulting in less accurate estimates of sleep parameters compared to objective measures (Fernandez-Mendoza et al., 2011; Silva et al., 2007). The current findings therefore offer valuable insight into the subjective experience of sleep from an adolescent perspective, with the potential for future work to combine the iNOD's insight alongside more objective, verifiable measures of social media activity and sleep parameters to build a more holistic understanding of adolescent sleep that unites physiology, behaviour and experience (Gregory \& Sadeh, 2012; Tubbs et al., 2019). 


\subsubsection{Conclusions}

This study provides a rigorously developed and validated new self-report measure that captures difficulty disengaging from social media at night: the index of Nighttime Offline Distress (iNOD). Its two subscales allow researchers and practitioners to move beyond measuring the frequency and duration of social media use, to instead meaningfully examine the extent to which individuals struggle to disconnect due to concerns voiced by adolescents around Staying Connected and Following Etiquette. By filling a gap in currently available measurement tools and capturing a novel construct, the INOD can support efforts to build more nuanced understanding of the sleep-specific implications of online social interactions for those navigating adolescence in today's 24/7 connected world. The current findings indicate that extended wakefulness in bed before attempting to sleep is typical for today's adolescents, reinforcing the importance of considering not only sleep onset latency but also shut eye latency (Exelmans \& Van den Bulck, 2017a). These results further indicate a fragmented process of sleep displacement for those who struggle to disconnect - and stay disconnected - from potential online interactions to allow an uninterrupted sleep opportunity. Whilst many adolescents do not experience considerable difficulties disengaging from social media at night, those who do struggle with these concerns tend to use social media for longer than intended, reporting shorter sleep duration and poorer sleep quality. Efforts to update sleep education and interventions should aim to target these underlying concerns about staying connected and following etiquette, rather than simply restricting social media or device access. 


\section{Acknowledgements}

The authors would like to thank the following individuals for their valued contributions, which included comments on candidate scale items, analytical approach and previous versions of this manuscript: Dr Liese Exelmans, Dr Maria Gardani, Dr Chiara Horlin, Prof Andrew Pzybylski, Prof Jan Van den Bulck and Dr Netta Weinstein.

Funding: This work was supported by the UK Economic and Social Research Council [grant number ES/J500136/1]. 


\section{References}

Althubaiti, A. (2016). Information bias in health research: Definition, pitfalls, and adjustment methods. Journal of Multidisciplinary Healthcare, 9, 211-217. doi: 10.2147/JMDH.S104807

Andreassen, C. S., Torsheim, T., Brunborg, G. S., \& Pallesen, S. (2012). Development of a Facebook addiction scale. Psychological Reports, 110(2), 501-517. doi:10.2466/02.09.18.pr0.110.2.501-517

Assunção, R. S., \& Matos, P. M. (2014). Perspectives of adolescents about Facebook use: A qualitative study. Psicologia em Estudo, 19, 539-547. doi:10.1590/141373722133716

Bartel, K., \& Gradisar, M. (2017). New directions in the link between technology use and sleep in young people. In S. Nevšímalová \& O. Bruni (Eds.), Sleep disorders in children (pp. 69-80). Cham, Switzerland: Springer International Publishing.

Bartel, K., Scheeren, R., \& Gradisar, M. (2019). Altering adolescents' pre-bedtime phone use to achieve better sleep health. Health Communication, 34(4), 456-462. doi:10.1080/10410236.2017.1422099

Bhat, S., Pinto-Zipp, G., Upadhyay, H., \& Polos, P. G. (2018). “To sleep, perchance to tweet": In-bed electronic social media use and its associations with insomnia, daytime sleepiness, mood, and sleep duration in adults. Sleep Health, 4(2), 166173. doi:10.1016/j.sleh.2017.12.004

Blakemore, S.-J. (2018). Avoiding social risk in adolescence. Current Directions in Psychological Science, 27(2), 116-122. doi:10.1177/0963721417738144

Blakemore, S.-J., \& Mills, K. L. (2014). Is adolescence a sensitive period for sociocultural processing? Annual Review of Psychology, 65, 187-207.

Browne, M. W., \& Cudeck, R. (1992). Alternative ways of assessing model fit. Sociological Methods \& Research, 21(2), 230-258. doi:10.1177/0049124192021002005

Cain, N., \& Gradisar, M. (2010). Electronic media use and sleep in school-aged children and adolescents: A review. Sleep Medicine, 11(8), 735-742. doi:10.1016/j.sleep.2010.02.006

Cain, N., Gradisar, M., \& Moseley, L. (2011). A motivational school-based intervention for adolescent sleep problems. Sleep Medicine, 12(3), 246-251. doi:10.1016/j.sleep.2010.06.008

Carr, C. T., \& Hayes, R. A. (2015). Social media: Defining, developing, and divining. Atlantic Journal of Communication, 23(1), 46-65. doi:10.1080/15456870.2015.972282

Carskadon, M. A. (2011). Sleep in adolescents: The perfect storm. Pediatric clinics of North America, 58(3), 637-647. doi:10.1016/j.pcl.2011.03.003

Cassoff, J., Rushani, F., Gruber, R., \& Knäuper, B. (2014). Evaluating the effectiveness of the motivating teens to sleep more program in advancing bedtime in adolescents:

A randomized controlled trial. BMC Psychology, 2(1), 6. doi:10.1186/2050-7283-2-6

Crowley, S. J., Wolfson, A. R., Tarokh, L., \& Carskadon, M. A. (2018). An update on adolescent sleep: New evidence informing the perfect storm model. Journal of Adolescence, 67, 55-65. doi:10.1016/j.adolescence.2018.06.001

DeVellis, R. F. (2016). Scale development: Theory and applications. Los Angeles, CA: SAGE Publications. 
Donnellan, M. B., Oswald, F. L., Baird, B. M., \& Lucas, R. E. (2006). The mini-ipip scales: Tiny-yet-effective measures of the big five factors of personality. Psychological Assessment, 18(2), 192-203. doi:10.1037/1040-3590.18.2.192

Ellison, N. B., Steinfield, C., \& Lampe, C. (2007). The benefits of Facebook "friends": Social capital and college students' use of online social network sites. Journal of Computer-Mediated Communication, 12(4). doi:10.1111/j.1083-6101.2007.00367.x

Espie, C. A., Kyle, S. D., Hames, P., Gardani, M., Fleming, L., \& Cape, J. (2014). The Sleep Condition Indicator: A clinical screening tool to evaluate insomnia disorder. BMJ Open, 4(3). doi:10.1136/bmjopen-2013-004183

Exelmans, L., Gradisar, M., \& Van den Bulck, J. (2018). Sleep latency versus shuteye latency: Prevalence, predictors and relation to insomnia symptoms in a representative sample of adults. Journal of Sleep Research, 27(6), e12737. doi:doi:10.1111/jsr.12737

Exelmans, L., \& Van den Bulck, J. (2017a). Bedtime, shuteye time and electronic media: Sleep displacement is a two-step process. Journal of Sleep Research, 26(3), 364370. doi:10.1111/jsr.12510

Fernandez-Mendoza, J., Calhoun, S. L., Bixler, E. O., Karataraki, M., Liao, D., VelaBueno, A., . . . Vgontzas, A. N. (2011). Sleep misperception and chronic insomnia in the general population: Role of objective sleep duration and psychological profiles. Psychosomatic Medicine, 73(1), 88-97. doi:10.1097/PSY.0b013e3181fe365a

Glorfeld, L. W. (1995). An improvement on Horn's parallel analysis methodology for selecting the correct number of factors to retain. Educational and Psychological Measurement, 55(3), 377-393. doi:10.1177/0013164495055003002

Gravetter, F., \& Wallnau, L. (2014). Essentials of statistics for the behavioural sciences (8th ed.). Belmont, CA: Wadsworth.

Gregory, A. M., \& Sadeh, A. (2012). Sleep, emotional and behavioral difficulties in children and adolescents. Sleep Medicine Reviews, 16(2), 129-136. doi:10.1016/j.smrv.2011.03.007

Hair, J. F., Tatham, R. L., Anderson, R. E., \& Black, W. (1998). Multivariate data analysis. Ohio, USA: Prentic Hall.

Haynes, W. (2013). Holm's method. In W. Dubitzky, O. Wolkenhauer, K.-H. Cho, \& H. Yokota (Eds.), Encyclopedia of systems biology (pp. 902-902). New York, NY: Springer New York.

Hogg, M. A., \& Reid, S. A. (2006). Social identity, self-categorization, and the communication of group norms. Communication Theory, 16(1), 7-30. doi:doi:10.1111/j.1468-2885.2006.00003.x

Holm, S. (1979). A simple sequentially rejective multiple test procedure. Scandinavian Journal of Statistics, 6(2), 65-70.

Holmgren, H. G., \& Coyne, S. M. (2017). Can't stop scrolling!: Pathological use of social networking sites in emerging adulthood. Addiction Research \& Theory, 25(5), 375382. doi:10.1080/16066359.2017.1294164

Horn, J. L. (1965). A rationale and test for the number of factors in factor analysis. Psychometrika, 30(2), 179-185. doi:10.1007/BF02289447

Hu, L. T., \& Bentler, P. M. (1999). Cutoff criteria for fit indexes in covariance structure analysis: Conventional criteria versus new alternatives. Structural Equation Modeling - a Multidisciplinary Journal, 6(1), 1-55. doi:10.1080/10705519909540118

Hubley, A. M. (2014). Discriminant validity. In A. C. Michalos (Ed.), Encyclopedia of quality of life and well-being research (pp. 1664-1667). Dordrecht, Netherlands: Springer. 
Jenkins-Guarnieri, M. A., Wright, S. L., \& Johnson, B. (2013). Development and validation of a social media use integration scale. Psychology of Popular Media Culture, 2(1), 38-50. doi:10.1037/a0030277

Kaiser, H. F. (1960). The application of electronic computers to factor analysis. Educational and Psychological Measurement, 20(1), 141-151. doi:10.1177/001316446002000116

Kline, P. (2000). The handbook of psychological testing. London: Routeledge.

Lee, F. S., Heimer, H., Giedd, J. N., Lein, E. S., Sestan, N., Weinberger, D. R., \& Casey, B. J. (2014). Adolescent mental health--opportunity and obligation. Science, 346(6209), 547-549. doi:10.1126/science. 1260497

Owens, J. (2014). Insufficient sleep in adolescents and young adults: An update on causes and consequences. Pediatrics, 134(3), E921-E932. doi:10.1542/peds.2014-1696

Pew Research Center. (May 2018). Teens, social media \& technology 2018. Retrieved from https: / /www.pewinternet.org/2018/05/31/teens-social-media-technology2018/

Pittman, M., \& Reich, B. (2016). Social media and loneliness: Why an Instagram picture may be worth more than a thousand Twitter words. Computers in Human Behavior, 62, 155-167. doi:10.1016/j.chb.2016.03.084

Przybylski, A. K., Murayama, K., DeHaan, C. R., \& Gladwell, V. (2013). Motivational, emotional, and behavioral correlates of fear of missing out. Computers in Human Behavior, 29(4), 1841-1848. doi:10.1016/j.chb.2013.02.014

R Core Team. (2018). R: A language and environment for statistical computing. Vienna, Austria: R Foundation for Statistical Computing. Retrieved from https: //www.Rproject.org/

Ross, C., Orr, E. S., Sisic, M., Arseneault, J. M., Simmering, M. G., \& Orr, R. R. (2009). Personality and motivations associated with Facebook use. Computers in Human Behavior, 25(2), 578-586. doi:10.1016/j.chb.2008.12.024

Scott, H., Biello, S. M., \& Woods, H. C. (2019). Identifying drivers for bedtime social media use despite sleep costs: The adolescent perspective. Sleep Health, 5(6), 539-545.

Scott, H., \& Woods, H. C. (2018). Fear of missing out and sleep: Cognitive behavioural factors in adolescents' nighttime social media use. Journal of Adolescence, 68, 6165. doi:10.1016/j.adolescence.2018.07.009

Scott, H., \& Woods, H. C. (2019). Understanding links between social media use, sleep and mental health: Recent proress and current challenges. Current Sleep Medicine Reports, 5(3), 141-149. doi:10.1007/s40675-019-00148-9

Seabrook, E. M., Kern, M. L., \& Rickard, N. S. (2016). Social networking sites, depression, and anxiety: A systematic review. JMIR Mental Health, 3(4), e50. doi: $10.2196 /$ mental. 5842

Sheldon, K. M., \& Bettencourt, B. A. (2002). Psychological need-satisfaction and subjective well-being within social groups. British Journal of Social Psychology, 41(1), 25-38. doi:doi:10.1348/014466602165036

Short, M. A., Weber, N., Reynolds, C., Coussens, S., \& Carskadon, M. A. (2018). Estimating adolescent sleep need using dose-response modeling. Sleep, 41(4), 114. doi:10.1093/sleep/zsy011

Silva, G. E., Goodwin, J. L., Sherrill, D. L., Arnold, J. L., Bootzin, R. R., Smith, T., . . . Quan, S. F. (2007). Relationship between reported and measured sleep times: The sleep heart health study (SHHS). Journal of Clinical Sleep Medicine, 3(6), 
The Guardian. (25 December 2016). Screen-based lifestyle harms children's health. https: / / www.theguardian.com/education/2016/dec/25/screen-based-lifestyleharms-health-of-children

The Lancet. (2019). Social media, screen time, and young people's mental health. The Lancet, 393(10172), 611. doi:10.1016/S0140-6736(19)30358-7

Tubbs, A. S., Dollish, H. K., Fernandez, F., \& Grandner, M. A. (2019). The basics of sleep physiology and behavior. In M. A. Grandner (Ed.), Sleep and health (pp. 3-10). London, United Kingdom: Academic Press.

UK House of Commons Science and Technology Committee. (2019). Impact of social media and screen-use on young people's health. https: / / publications.parliament.uk/pa/cm201719/cmselect/cmsctech/822/82202. htm

Van den Bulck, J. (2004). Television viewing, computer game playing, and internet use and self-reported time to bed and time out of bed in secondary-school children. Sleep, 27(1), 101-104.

van den Eijnden, R. J. J. M., Lemmens, J. S., \& Valkenburg, P. M. (2016). The Social Media Disorder Scale. Computers in Human Behavior, 61, 478-487. doi:10.1016/j.chb.2016.03.038

Watson, J. C. (2017). Establishing evidence for internal structure using exploratory factor analysis. Measurement and Evaluation in Counseling and Development, 50(4), 232238. doi:10.1080/07481756.2017.1336931

Wilcox, R. (2012). Comparing two groups. In R. Wilcox (Ed.), Introduction to robust estimation and hypothesis testing (3rd ed., pp. 137-213). Boston, MA: Academic Press.

Woods, H. C., \& Scott, H. (2016). \#sleepyteens: Social media use in adolescence is associated with poor sleep quality, anxiety, depression and low self-esteem. Journal of Adolescence, 51, 41-49. doi:10.1016/j.adolescence.2016.05.008 